\title{
Proof-of-Concept of a Novel \\ Micro-Bioreactor for Fast Development of Industrial Bioprocesses
}

\author{
N. Reis, C.N. Gonçalves, A.A. Vicente, J.A. Teixeira \\ Centro de Engenharia Biológica, Universidade do Minho, Campus de Gualtar, \\ 4710-057 Braga, Portugal; telephone: +351.253.604400; fax: +351.253.678986; \\ e-mail: nunoreis@deb.uminho.pt
}

Received 20 February 2006; accepted 16 May 2006

Published online 6 June 2006 in Wiley InterScience (www.interscience.wiley.com). DOI: 10.1002/bit.21035

\begin{abstract}
The experimental performance of a novel micro-bioreactor envisaged for parallel screening and development of industrial bioprocesses has been tested in this work. The micro-bioreactor with an internal volume of $4.5 \mathrm{~mL}$ is operated under oscillatory flow mixing (OFM), where a controllable mixing and mass transfer rates are achieved under batch or continuous laminar flow conditions. Several batch fermentations with a flocculent Saccharomyces cerevisiae strain were carried out at initial glucose concentrations $\left(S_{0}\right)$ range of $\sim 5-20 \mathrm{~g} / \mathrm{L}$ and compared to yeast growth kinetics in a stirred tank (ST) bioreactor. Aerobic fermentations were monitored ex situ in terms of $\mathrm{pH}, \mathrm{DO}$, glucose consumption, and biomass and ethanol production (wherever applicable). An average biomass production increase of $83 \%$ was obtained in the micro-bioreactor when compared with the ST, with less $93.6 \%$ air requirements. It also corresponded to a $214 \%$ increase on biomass production when compared with growth in a shaken flask (SF) at $S_{0}=20 \mathrm{~g} / \mathrm{L}$. Further anaerobic fermentations at the same initial glucose concentration ranges gave the opportunity to use state-ofthe-art fiber optics technology for on-line and real-time monitoring of this bioprocess. Time profiles of biomass concentration (measured as optical density (OD)) were very similar in the ST bioreactor and in the micro-bioreactor, with a highly reproducible yeast growth in these two scale-down platforms. (c) 2006 Wiley Periodicals, Inc.

Keywords: novel micro-bioreactor; scale-down; oscillatory flow mixing; biomass production; fermentations; Saccharomyces cerevisiae
\end{abstract}

\section{INTRODUCTION}

High-throughput techniques are a major support for bioprocess development. As the number of experimental parameters (environmental and culture conditions) to be optimized is vast a significant number of fermentations must be carried out. The characteristics of the currently available equipment make this procedure labor intensive and costly meaning a waste of valuable capital and time resources (Kostov et al., 2001).

Correspondence to: N. Reis

Contract grant sponsor: Fundação para a Ciência e a Tecnologia

Contract grant number: SFRH/BD/6954/2001
This reasoning is adequate both for shake flasks and fully equipped standard laboratory bioreactors (Tholudur et al., 1999). Obviously, shake flasks due to its simplicity and low cost, are the most currently used pieces of equipment. However, its limitations concerning process optimization are clear as they present a reduced capacity for controlling the process parameters (Buchs, 2001; Maier and Buchs, 2001; Rhodes and Gaden, 1957). On the other hand, although allowing for a precise monitoring of bioprocess parameters, standard laboratory bioreactors represent a high capital cost if several fermentations are to be carried out in a short period of time (in parallel). Thus, a technology for fast, reliable, and inexpensive parallel bioprocessing is strongly desirable. The idea may be to scale-down the volume of a bioreactor system while preserving its control capabilities.

Recently, plate readers facilitated the parallel studies in bioprocesses ( $\mathrm{Li}$ et al., 2000), but the on-line information outcoming from a plate reader is limited to one or two parameters (e.g., absorbance and/or fluorescence), because they are not equipped with chemical sensors or actuators for bioprocess control. The automated shaken microwell system has been relevant in obtaining information related with bacterial fermentations (Duetz and Witholt, 2001; Duetz et al., 2000), animal cell cultures (Girard et al., 2001), and other biotransformations (Doig et al., 2002; Weiss et al., 2002). However, as indicated by the limited data available, the volumetric mass transfer coefficient in a shaken microwell (Weiss et al., 2002) is lower at least by a factor of 10 , compared to a conventional scale fermenter.

Walther et al. (1994) have described a miniature bioreactor with a working volume of $3 \mathrm{~mL}$ for cell culture in a space laboratory. Later on, Kostov et al. (2001) introduced the design of a $2 \mathrm{~mL}$ working volume micro-bioreactor provided with an optical sensing system. An E. coli fermentation in both the micro-bioreactor and a standard 1-L bioreactor showed similar $\mathrm{pH}$, dissolved oxygen, and optical density (OD) profiles. Mixing was achieved by the action of a magnetic stirrer placed at the bottom of the well. Girard et al. (2001) also presented a small-scale bioreactor system for process development and optimization, based on agitated (by 
a rotational shaker) 12-well microtiter plates with a working volume of $2 \mathrm{~mL}$. Such reactor improved maximum mammalian cells (CHO and HEK 293 cell lines) density and $\mathrm{pH}$ stability. Its automation and application to high throughputs is possible by running several hundred small-scale bioreactor experiments in parallel. Lamping et al. (2003) also presented the design of a new miniature bioreactor with a diameter equal to that of a single well of a 24-well plate, for high throughput automated bioprocessing. Mixing was provided by a set of three impellers mechanically driven via a microfabricated electric motor and aeration was achieved with a single tube sparger. Such design features resulted in measured volumetric mass transfer coefficients in the miniature reactor in the range $100-400 / \mathrm{h}$, typical of those reported for large-scale fermentation. Fiber optic probes were applied to the continuous monitoring of dissolved oxygen tension and cell biomass concentration during fermentations of E. coli. More recently, Doig et al. (2005a,b) presented a novel miniaturized bubble column bioreactor for high throughput cell cultivation. The miniature bioreactor $(2 \mathrm{~mL})$ consists of static deep well microtiter plate. Air was supplied to each well assuring a volumetric mass transfer coefficient for oxygen, $k_{L} a$, of up to $220 / \mathrm{h}$ and was used to optimize the growth conditions of Bacillus subtilis, a strict aerobic microorganism. The system was not shaken and allowed installation of miniature optical probes.

As previously pointed out, bioprocess development requires an accurate and on line measurement of key parameters. This can be easily carried out at lab scale in standard bioreactors. However, the measuring techniques available for these systems cannot be applied in a straightforward manner to small volume systems. Being so, innovative techniques are required as is the case of using optical probes (e.g., Lamping et al., 2003).

When the yeast Saccharomyces cerevisiae is grown in a batch culture under aerobic conditions with glucose as sole energy and carbon source, ethanol can be released into the culture liquid as a by-product. In a first growth phase, biomass is formed and ethanol is accumulated at the outlay of glucose consumption. The ethanol is assimilated only in the subsequent growth phase, which begins after glucose is completely exhausted and the cells have adapted to the new carbon source (Rieger et al., 1983; Sonnleitner and Kappeli, 1986). The model for yeast growth presented by Sonnleitner and Kappeli (1986) considers three pure metabolic routes involved in (aerobic and anaerobic) glucose breakdown: (i) oxidative and (ii) reductive glucose catabolism as well as (iii) ethanol utilization. Under anaerobic conditions, the glucose reduction (fermentation) is the only mode of energy production but alcoholic fermentation may occur even under aerobic conditions (Vandijken and Scheffers, 1986) if the glucose concentration surpasses a critical threshold value (Sonnleitner and Kappeli, 1986; Verduyn et al., 1984).

Cell cultures studies in conventional oscillatory flow reactors (OFRs) were limited to the experimental determination of mass transfer of oxygen into a yeast suspension. $\mathrm{Ni}$ et al. (1995) reported the study of ressuspended S. cerevisiae (from frozen baker's) in an aerated 50-mm internal diameter OFR with an $11 \%$ increase in the $k_{L} a$, compared to those obtained in a ST. Such trends in $k_{L} a$ were consistent with the fluid mechanics observed within both systems. Lee et al. $(2001,2002)$ considered the OFR a viable reactor for protein refolding via direct dilution; as the mixing characteristics of the OFR are well described and controllable (e.g., Mackley, 1991) and also suggested that OFR can be scaled-up to process scale without loss of mixing efficiency.

In this article the proof-of-concept of a novel microbioreactor recently designed (Reis et al., 2005) is presented buy running several batch fermentations with a flocculent $S$. cerevisiae strain, both under aerobic and anaerobic conditions. The objective is to show that the novel reactor can be used with advantages over the more traditional SF and ST bioreactors. The possibility of using fiber optical technology for on-line and real-time monitoring of the bioprocesses going on the reactor has also been demonstrated by monitoring the biomass concentration through OD measurements.

\section{MATERIALS AND METHODS}

The proof-of-concept has been established by carrying out fermentations in the micro-bioreactor system using as workhorse a flocculent $S$. cerevisiae strain at four different initial glucose concentrations in the range of $\sim 5-20 \mathrm{~g} / \mathrm{L}$, under aerobic and anaerobic growth conditions. The results were compared with those from fermentations in a lab-scale stirred tank (ST) bioreactor.

\section{Micro-Bioreactor Geometry and Operation}

The novel micro-bioreactor is composed of a $4.4 \mathrm{~mm}$ internal diameter jacketed-glass tube provided with smooth periodic constrictions (SPC tube) and a volume of approx. $4.5 \mathrm{~mL}$ (Fig. 1). The fluid is oscillated at the bottom of a SPC tube at a certain oscillation frequency, $f$, and amplitude (center-topeak), $x_{0}$. An effective mixing is achieved by generating, expanding and transporting vortex rings, through the use of oscillatory flow mixing (OFM) technology (e.g., Mackley, 1991). A wide range of mixing intensities can be achieved simply by changing $f$ and/or $x_{0}$. OFM regimes within the micro-bioreactor have been previously identified (Reis et al., 2004b, 2005).

This novel micro-bioreactor was designed to offer sterile conditions with sufficient mass-transfer capability so as to support, for example, the fermentation of commercially important recombinant organisms (Reis et al., 2004a). Each SPC tube is temperature controlled and it can be coupled to a fiber optics system to achieve online monitoring and control of, for example, OD, DO, or $\mathrm{pH}$.

In this work the micro-bioreactor was operated as follows: a single sterilized (at $110^{\circ} \mathrm{C}$ for $40 \mathrm{~min}$ ) SPC tube $(4.5 \mathrm{~mL}$ operating volume) was positioned vertically (Fig. 1), and the fluid oscillated at a $f$ and $x_{0}$ of $18 / \mathrm{s}$ and $3 \mathrm{~mm}$, respectively, where the flow is turbulent like (Reis et al., 2005). In aerobic 


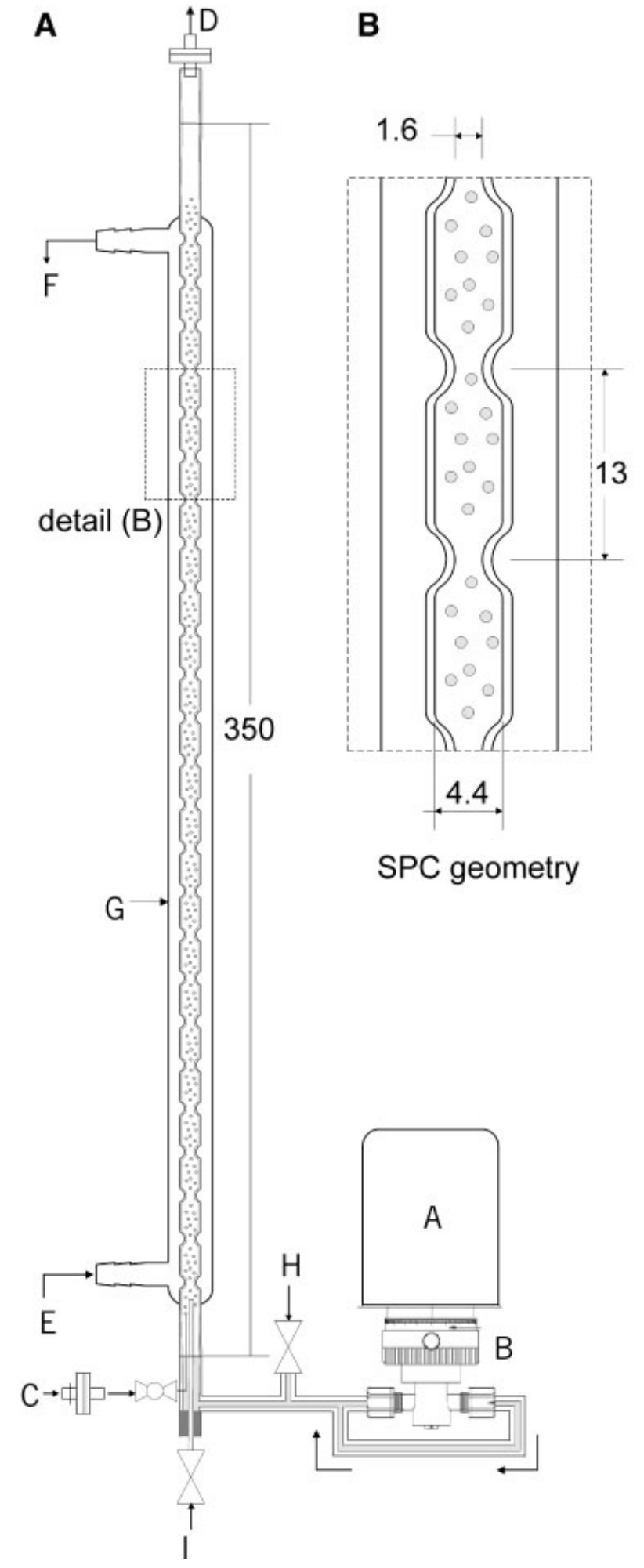

Figure 1. A: Experimental setup used in batch fermentations of S. cerevisiae: A, rotary motor; B, piston pump; C, gas inlet; D, gas outlet; E, fluid heating inlet; F, fluid heating outlet; G, SPC tube; H, purging port; I, sampling port. B: Detail of smooth periodic constricted (SPC) tube geometry, which composes the novel, designed oscillatory flow micro-bioreactor. All dimensions are in $\mathrm{mm}$. yeast growth experiments, the reactor was continuously aerated through the bottom (Fig. 1A) with sterile air at an average superficial gas velocity, $U_{\mathrm{g}}=0.37 \mathrm{~mm} / \mathrm{s}$ (equivalent to a gas flow rate of $0.064 \mathrm{vvm}$, where vvm is the volume of gas-phase per volume of reactor per minute). The aeration rate was controlled using a needle micro valve. In all fermentations, the temperature was maintained at $25^{\circ} \mathrm{C}$ by circulating water at that temperature through the jacket of the SPC tube.

\section{5-L ST Bioreactor Geometry}

A 5-L ST bioreactor-Model microDCU system, installed with Twin and MCU-200 controllers (B. Braun Biotech International, Melsungen, Germany) with a working volume of $4.7 \mathrm{~L}$ was used in the aerobic fermentations of $S$. cerevisiae. The ST bioreactor was sterilized at $121^{\circ} \mathrm{C}$ during 30 min with $4.23 \mathrm{~L}$ of YPD medium. After stabilization of temperature and dissolved oxygen, it was inoculated with the $470 \mathrm{~mL}$ seed culture. The ST bioreactor was aerated with sterile air at an aeration rate of $1.1 \mathrm{vvm}$ and the agitation speed was controlled at $150 \mathrm{rpm}$. The aeration rate was controlled using a Hastings (Hampton, Virginia) mass-flow controller. A minimum $\mathrm{O}_{2}$ concentration of $30 \%$ was assured throughout the fermentation by means of a cascade controller using aeration as master and agitation as slave controller. $\mathrm{pH}$ was analyzed on-line using a $\mathrm{pH}$ probe connected to the microDCU system.

\section{2-L ST Bioreactor Geometry}

A 2-L ST bioreactor-Model Biostat M (B. Braun Biotech International) with a working volume of $1.5 \mathrm{~L}$ was used for yeast growth at anaerobic conditions. The ST bioreactor's agitation speed was set to $150 \mathrm{rpm}$ and the reactor containing $1.5 \mathrm{~L}$ of YPD medium was sterilized at $121^{\circ} \mathrm{C}$ during $20 \mathrm{~min}$.

\section{Aerobic Growth of S. cerevisiae}

S. cerevisiae yeast was selected due to its high industrial relevance (Kappeli, 1986). S. cerevisiae RIBM 655 was preserved at $4^{\circ} \mathrm{C}$ on YPD (10 g/L yeast extract (Merck Co., Darmstadt, Germany), $20 \mathrm{~g} / \mathrm{L}$ peptone (Bacto $^{\mathrm{TM}}$ peptone, Quilaban, Le Pont de Claix, France), and 20 g/L D-glucose anhydrous (HiMedia Laboratories, Pvt. Ltd., Mumbai, India) agar slants. One agar slant was washed with sterile YPD medium and the obtained cell suspension was transferred to a $1000 \mathrm{~mL}$ Erlenmeyer flask (working volume of $470 \mathrm{~mL}$ ). That culture was then incubated overnight at $27^{\circ} \mathrm{C}$ (Certomat ${ }^{\mathrm{R}} \mathrm{H}$ ) under orbital shaking at $150 \mathrm{rpm}\left(\right.$ Certomat $^{\mathrm{R}} \mathrm{R}$ ) (both from B. Braun Biotech International). Cells for the inoculation of the SF, the 5-L ST bioreactor and the micro-bioreactor were harvested at the end of the exponential growth phase (after 16-17 h from inoculation), which was estimated by measuring the OD of a sample at $\lambda=620 \mathrm{~nm}$. Four different initial glucose concentrations (ranging from 
5 to $20 \mathrm{~g} / \mathrm{L}$ ) were tested in both the 5-L ST bioreactor and in the micro-bioreactor (Table I).

Fermentation temperature was controlled at $25^{\circ} \mathrm{C}$. To avoid foaming formation due to mixing $1 \mathrm{ppm}$ of antifoam $\mathrm{O}$ 30 (Sigma Aldrich, Steinheim, Germany) was added to the fermentation media in the micro-bioreactor.

Samples were collected from each fermentation vessel (5L ST bioreactor, SF, and micro-bioreactor) at every hour and centrifuged $15,000 \mathrm{rpm}$ for $10 \mathrm{~min}$ (centrifuge model Sigma 112, B. Braun Biotech International). The precipitate was afterwards used for determining the biomass by spectrophotometry, while the supernatant (from 5-L ST bioreactor and micro-bioreactor) was used for determination of the glucose and ethanol by HPLC.

\section{Biomass Determination}

The precipitate from each centrifuged sample was treated with de-flocculation solution (washed three times with $\mathrm{NaCl}$ $1.5 \%$ (Merck Co.), $\mathrm{pH}$ adjusted to 3.0 with $\mathrm{HCl}$ ). After ressuspension in de-flocculation solution, the OD was measured at $620 \mathrm{~nm}$ using a Jasco's V-560 spectrometer (Jasco, Tokyo, Japan). The cell dry weight was obtained from a calibration curve, which was found linear up to cell dry weights $(X)$ of $330 \mathrm{mg} / \mathrm{L}$, corresponding to cell turbidity (absorbance) of 0.802 at $620 \mathrm{~nm}$.

\section{Glucose and Ethanol Determination}

Glucose and ethanol concentrations were determined using a HPLC system (830-RI detector, pump 830-PU, both from Jasco), installed with a Chrompack Organic Acids Column (Varian, Inc., Palo Alto, CA). The mobile phase was $\mathrm{H}_{2} \mathrm{SO}_{4}$

Table I. Averaged yields of biomass on substrate $\left(Y_{X / S}\right)$ and specific substrate uptake rate $\left(q_{s}=\mu / Y_{\mathrm{X} / S}\right)$ during the exponential phase of the aerobic growth of $S$. cerevisiae in bioprocesses I-IV and in three different small-scale vessels: 5-L stirred tank (ST) bioreactor, micro-bioreactor and shake flask (SF).

\begin{tabular}{|c|c|c|c|c|}
\hline & Bioprocess & $S_{0}[\mathrm{~g} / \mathrm{L}]$ & $\begin{array}{c}Y_{X / S} \\
{\left[g_{\text {cells }} /\right.} \\
\left.g_{\text {glucose }}\right]\end{array}$ & $\begin{array}{c}q_{\mathrm{s}}\left[\mathrm{g}_{\text {glucose }} /\right. \\
\left.\mathrm{g}_{\text {cells }} / \mathrm{h}\right]\end{array}$ \\
\hline \multirow[t]{4}{*}{ 5-L ST bioreactor ${ }^{\mathrm{a}}$} & Ia & 4.5 & 0.27 & -1.10 \\
\hline & IIa & 9.6 & 0.27 & -0.98 \\
\hline & IIIa & 15.0 & 0.20 & -1.59 \\
\hline & IVa & 17.8 & 0.26 & -1.06 \\
\hline \multirow[t]{4}{*}{ Micro-bioreactor $^{\mathrm{a}}$} & $\mathrm{Ib}$ & 4.2 & 0.36 & -0.91 \\
\hline & $\mathrm{IIb}$ & 8.0 & 0.54 & -0.64 \\
\hline & $\mathrm{IIIb}$ & 12.0 & 0.42 & -0.68 \\
\hline & $\mathrm{IVb}$ & 19.0 & 0.40 & -0.65 \\
\hline Shake flask ${ }^{b}$ & IVc & 20.0 & 0.16 & - \\
\hline \multirow[t]{2}{*}{ Literature } & & $40.0^{c}$ & $0.11^{\mathrm{c}}$ & - \\
\hline & & - & $0.50^{\mathrm{d}}$ & - \\
\hline
\end{tabular}

$S_{0}$ is the initial glucose concentration, as measured after inoculation with $10 \% \mathrm{v} / \mathrm{v}$ of seed culture.

${ }^{\text {a }}$ Experimental results from aerobic growth at $25^{\circ} \mathrm{C}$ inoculated with $10 \%$ seed culture.

${ }^{\mathrm{b}}$ Results from seed culture kinetics at $27^{\circ} \mathrm{C}$ and shaking at $150 \mathrm{rpm}$.

${ }^{\mathrm{c}}$ Data from Haack et al. (2004).

${ }^{\mathrm{d} D a t a}$ from Pamment et al. (1978).
$(0.01 \mathrm{~N})$ at a flow rate of $0.6 \mathrm{~mL} / \mathrm{min}$. Temperature was maintained at $60^{\circ} \mathrm{C}$ with an oven Chrompack column thermostat (Varian Inc., Palo Alto, CA). Under these conditions, distinct peaks of glucose and ethanol were resolved within $20 \mathrm{~min}$. The mean retention time was 7.17 $( \pm 0.03) \mathrm{min}$ for glucose and $15.38( \pm 0.07) \mathrm{min}$ for ethanol.

\section{Anaerobic Growth of $S$. cerevisiae}

S. cerevisiae RIBM 655 was preserved at $4{ }^{\circ} \mathrm{C}$ in a water solution. Cells were ressuspended and then transferred to a small container. Apart, in a 5-L Erlenmeyer, sterilized YPD medium (10 g/L yeast extract (Merck Co.), $20 \mathrm{~g} / \mathrm{L}$ peptone (Bacto $^{\mathrm{TM}}$ peptone, Quilaban), and $20 \mathrm{~g} / \mathrm{L}$ D-glucose anhydrous (HiMedia Laboratories, Pvt. Ltd) previously degassed for 30 min with $\mathrm{N}_{2}$ was inoculated with $\sim 10 \%$ (v/v) of ressuspended cells such that the turbidity (OD measured with a V-560 spectrometer (Jasco) at $620 \mathrm{~nm}$ ) was $\sim 0.5$. Finally, this 'initial culture' was aseptically transferred (pumped) to the fermentation vessels (2-L ST bioreactor and micro-bioreactor) with the desired volumes. The fermentation temperature was controlled at $25^{\circ} \mathrm{C}$ and $\mathrm{pH}$ was not controlled. Four different initial glucose concentrations were tested: $5,10,15$, and $20 \mathrm{~g} / \mathrm{L}$.

\section{Monitoring of Medium OD Along the Anaerobic Growth}

OD of cell growth media was on-line and real-time measured in the 2-L ST bioreactor and in the micro-bioreactor by means of reflectance optical micro-probes connected to a multi-channel optic spectrometer system (Avantes, Eerbeek, The Netherlands). Due to the different vessel geometries, two different probes were used. In the micro-bioreactor, a reflection probe (FCR-7UV200-1.5x100-2) with a small tip $(1.5 \mathrm{~mm})$ was installed in the top, in contact with the fermentation media. In the 2-L ST bioreactor, a standard reflection probe provided with a mirror at $45^{\circ}$ (FCR7UV200-2-45-ME) was installed inside through the top. In both cases, great attention was taken with probes installation, namely to avoid contact with light's reflectance surfaces (walls or impellers). Reading of the light coming from both probes was made on-line and simultaneously using a fourchannel optical spectrometer AvaLights-2048. The CCD detector was connected to an electronic board with 14 bit AD converter and USB/RS-232 interface. Data transfer between the optic spectrometer and a personal computer was controlled by AvaSoft full software. A tungsten halogen light source (AvaLight-HAL) was connected to FCR-7UV200-245-ME while a $475 \mathrm{~nm}$ LED light source (AvaLigh-LED475) was attached to FCR-7UV200-1.5x100-2. Thus, the integrals of the measured medium absorbance between 610 and $630 \mathrm{~nm}$ (for FCR-7UV200-1.5x100-2) and 465 and $485 \mathrm{~nm}$ (for FCR-7UV200-2-45-ME) were ten times averaged and recorded at every 5 min using suitable integration times. The measured OD (absorbance) values were found to change linearly with the dry cell weight in all 
experiments (results not shown). Being absorbance a logarithmic function of an amount, $I_{0} / I$ (where $I_{0}$ and $I$ are the reference and the sample reflected light, respectively), which is proportional to the quantity of biomass in the medium, this means that the function 'OD versus time' is a direct measure of the exponential growth phase, being its slope proportional to the specific growth rate $(\mu)$.

\section{RESULTS AND DISCUSSION}

\section{Aerobic Growth of S. cerevisiae on Glucose}

The effects of $S_{0}$ on the yeast growth metabolism at aerobic growth conditions were investigated in the range of $\sim 5-$ $20 \mathrm{~g} / \mathrm{L}$ (see Table I). Several experiments were performed in a 5-L ST bioreactor and in the micro-bioreactor. All experiments were run under well-controlled conditions of temperature, agitation, and aeration, as previously defined in Materials and Methods. Variations in biomass, glucose, and ethanol concentrations of the strain during the fermentation time were monitored ex situ. The end of fermentations was identified by the stabilization of biomass curve and a $\mathrm{pH}$ increase (results not shown). Once the fermentation was stopped, these observations were confirmed with the determination of substrate (glucose) concentration in the collected samples, demonstrating the effective glucose depletion. Figure 2 shows an example of the time course of biomass and ethanol productions using glucose as the sole substrate at ca. $15 \mathrm{~g} / \mathrm{L}$, in 5-L ST and in micro-bioreactor vessels. The higher biomass production (equal to $X-X_{0}$, where $X_{0}$ is the initial biomass concentration) achieved with the novel micro-bioreactor can be clearly observed, especially when taking into account the lower $S_{0}$ in the micro-bioreactor at the start of batch culture. In particular, this means the yield of biomass growth on glucose is enhanced in the micro-bioreactor.
In both culture vessels (Fig. 2), the kinetics of glucose consumption and biomass and ethanol production followed the expected patterns for these fermentation conditions. In the initial stage of fermentation, the glucose consumption was generally low due to the initial low cell density, but glucose concentration markedly decreased during batch fermentation after 200-400 min for both fermentation vessels. The resulting cell growth curve obtained from batch experiments has a typical sigmoidal shape (S-shape). The glucose consumption kinetics is apparently similar in both fermentation vessels. However very different biomass amounts were measured at the end of the fermentation: a $79 \%$ higher biomass production was obtained in the microbioreactor in comparison with the biomass production in the 5-L ST bioreactor. Such biomass enhancement reveals a higher yield of biomass on glucose in the micro-bioreactor, suggesting that the growth of $S$. cerevisiae on glucose in this reactor is mainly through the oxidative (respiratory) metabolic pathway, accordingly the model of Sonnleitner and Kappeli (1986). Glucose inhibits the uptake of ethanol as a substrate for growth while present in measurable concentrations, thus growth on ethanol is not considered. Because the oxidative glucose catabolism is a respiratory process (oxygen dependent), presumably such improvement in biomass production is due to the higher mass transfer rate of oxygen achieved with this novel micro-bioreactor, as shown by Reis et al. (2004a).

A comparison of the dry cell weight concentration obtained under aerated conditions is shown in Figure 3A (ST) and Figure 3B (micro-bioreactor), for bioprocesses I-IV. For the three higher initial glucose concentrations (bioprocesses II-IV), it is possible to observe the higher (up to $79 \%$ ) biomass production obtained with the microbioreactor, when compared to that produced in the 5-L ST bioreactor. In general, the biomass exponential growth phase was time-extended in the micro-bioreactor. The consequence
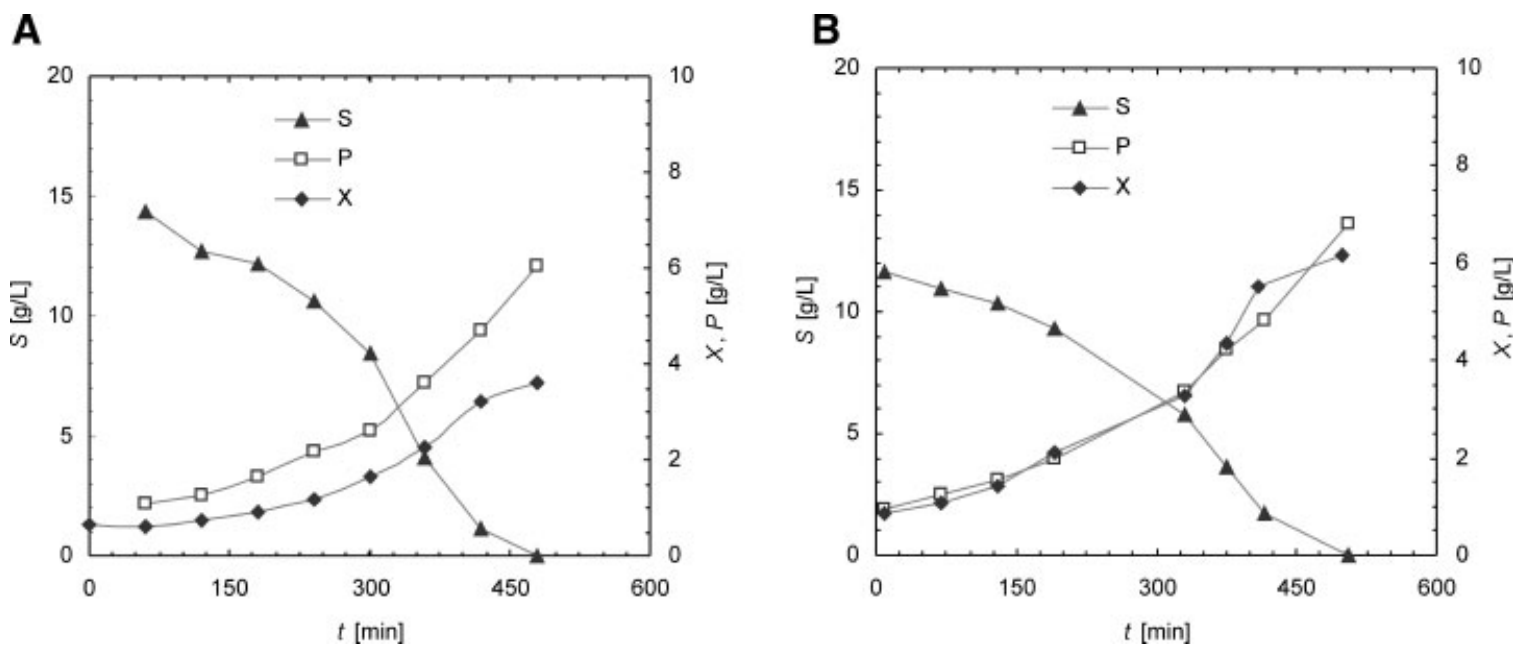

Figure 2. Time course of glucose concentration $(S)$, cell dry weight $(X)$, and ethanol concentration $(P)$ in batch aerobic-growth on glucose of S. cerevisiae (bioprocess IIIa and IIIb-see Table I). Fermentations in the 5-L stirred tank (ST) bioreactor (A), with an aeration rate of 1.1 vvm and in the micro-bioreactor $(\mathbf{B})$ with an aeration rate of $0.064 \mathrm{vvm}$. 
is a $70 \%$ and a $203 \%$ increase of biomass production in the ST and micro-bioreactor, respectively, as compared to the SF (Fig. 3C). Figure 3 presents a comparison of time profiles of dry cell weight in aerated vessels (A and B) with the micro-
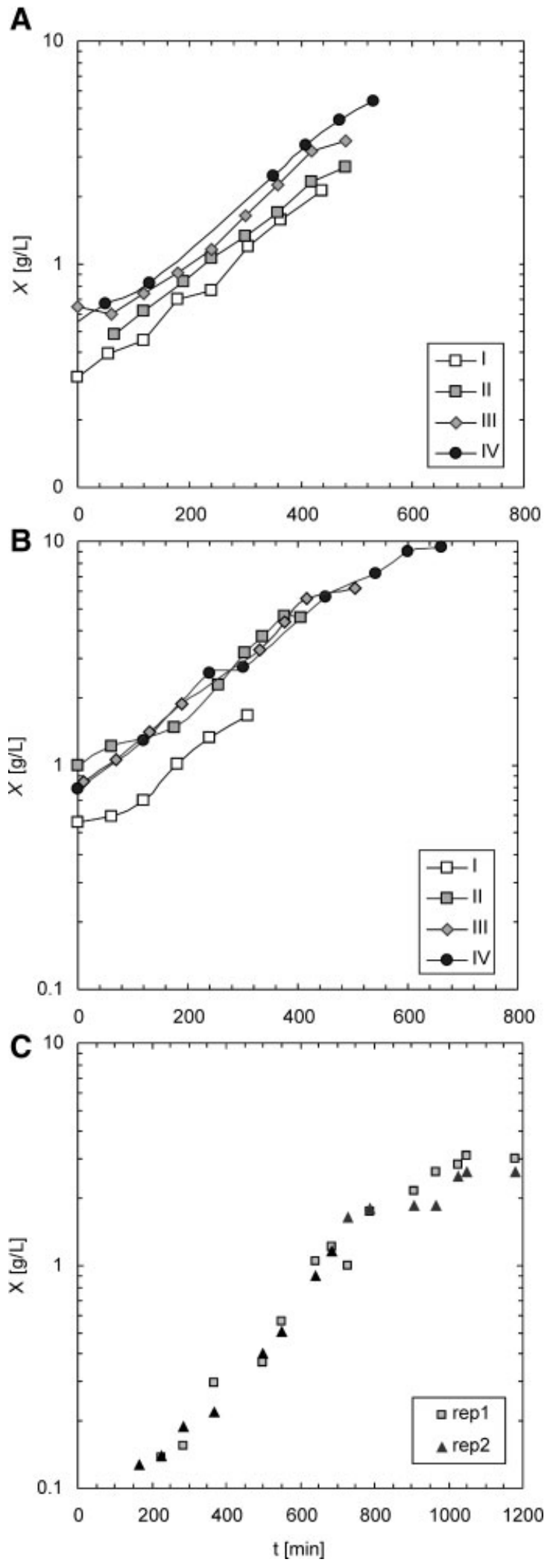

Figure 3. aerobic growth in a SF at $27^{\circ} \mathrm{C}$ (C) and also confirms the exponential phase of seed cultures after $16 \mathrm{~h}$ (the overnight incubation time).

Residual glucose concentrations along the batch growth are summarized in Figure 4 for both the 5-L ST bioreactor and the micro-bioreactor. The biomass (Fig. 3) and ethanol (results not shown) production were found to cease as the glucose concentration reaches exhaustion (Fig. 4), and therefore biomass and ethanol production were consistent with the time period of glucose catabolism in both reactor systems.

$\mathrm{pH}$ was not controlled during batch growth but it was monitored (results not shown) being above 5.0 in all the experiments with either ST, SF, and micro-bioreactor systems. At such $\mathrm{pH}$ values typically both cell growth and ethanol productivity are not inhibited (Yu and Zhang, 2004).

\section{Modeling the Aerobic Batch Growth of S. cerevisiae on Glucose}

\section{Yeast Growth Kinetics}

The kinetics involved in the batch growth of $S$. cerevisiae strain was characterized by its specific growth rate $(\mu)$, as suggested by Marin (1999). Kinetic studies have demonstrated that growth depends on the concentration of nutrients in the medium (see e.g., the review of Marin (1999)) but it was concluded that substrate concentration is not growth limiting in the tested range of concentrations $(\sim 5-20 \mathrm{~g} / \mathrm{L})$ for this $S$. cerevisiae strain (Fig. 5), where all the experiments showed an $\mu$ around an average value of $0.303 \pm 0.040 / \mathrm{h}$ for the micro-bioreactor, and $0.288 \pm 0.025 / \mathrm{h}$ for the 5 -L ST bioreactor. For the SF, an average growth rate of $0.268 / \mathrm{h}$ was calculated for a $S_{0}$ of $20 \mathrm{~g} / \mathrm{L}$, which is in agreement with the other systems' results. These $\mu$ values are smaller than that reported by Beck and Vonmeyen (1968) $(\mu=0.42$ for $S_{0}=9.2 \mathrm{~g} / \mathrm{L}$ ), but in the last case the fermentation conditions were controlled to $\mathrm{pH}=5.5$ and $30^{\circ} \mathrm{C}$, thus favoring the yeast growth.

The increase of the dry cell weight $\left(X-X_{0}\right)$ of $S$. cerevisiae was correlated with the initial glucose concentration $S_{0}$, as shown in Figure 6. The correlation slope gives an averagedglobal yield coefficient $\Delta X / \mathrm{S}_{0}$, which revealed that biomass production in the $S_{0}$ range of $\sim 5-20 \mathrm{~g} / \mathrm{L}$ is increased by $83 \%$ and $214 \%$ in the micro-bioreactor in comparison with the $5-\mathrm{L}$ $\mathrm{ST}$ and the SF, respectively. Substrate degradation during the exponential growth is therefore mostly achieved via respiratory metabolism instead of fermentative pathway in

Figure 3. Time profiles of cell dry weight, $X$ (log scale) in aerobic-batch glucose-growth of $S$. cerevisiae (bioprocesses I-IV). Fermentations in the 5-L ST bioreactor $(\mathbf{A})$ and in the micro-bioreactor $(\mathbf{B})$ with an aeration rate of $1.1 \mathrm{vvm}$ for the 5-L ST and $0.064 \mathrm{vvm}$ for the micro-bioreactor. C: Time profiles of dry cell weight in two replicates of $S$. cerevisiae growth in a shake flask (SF) starting with a glucose concentration of $20 \mathrm{~g} / \mathrm{L}$ (bioprocess IVc_-see also Table I); yeast was cultivated at $27^{\circ} \mathrm{C}$ and agitated in an orbital shaker at $150 \mathrm{rpm}$ (these experiments correspond to the seed culture's growth). 
A
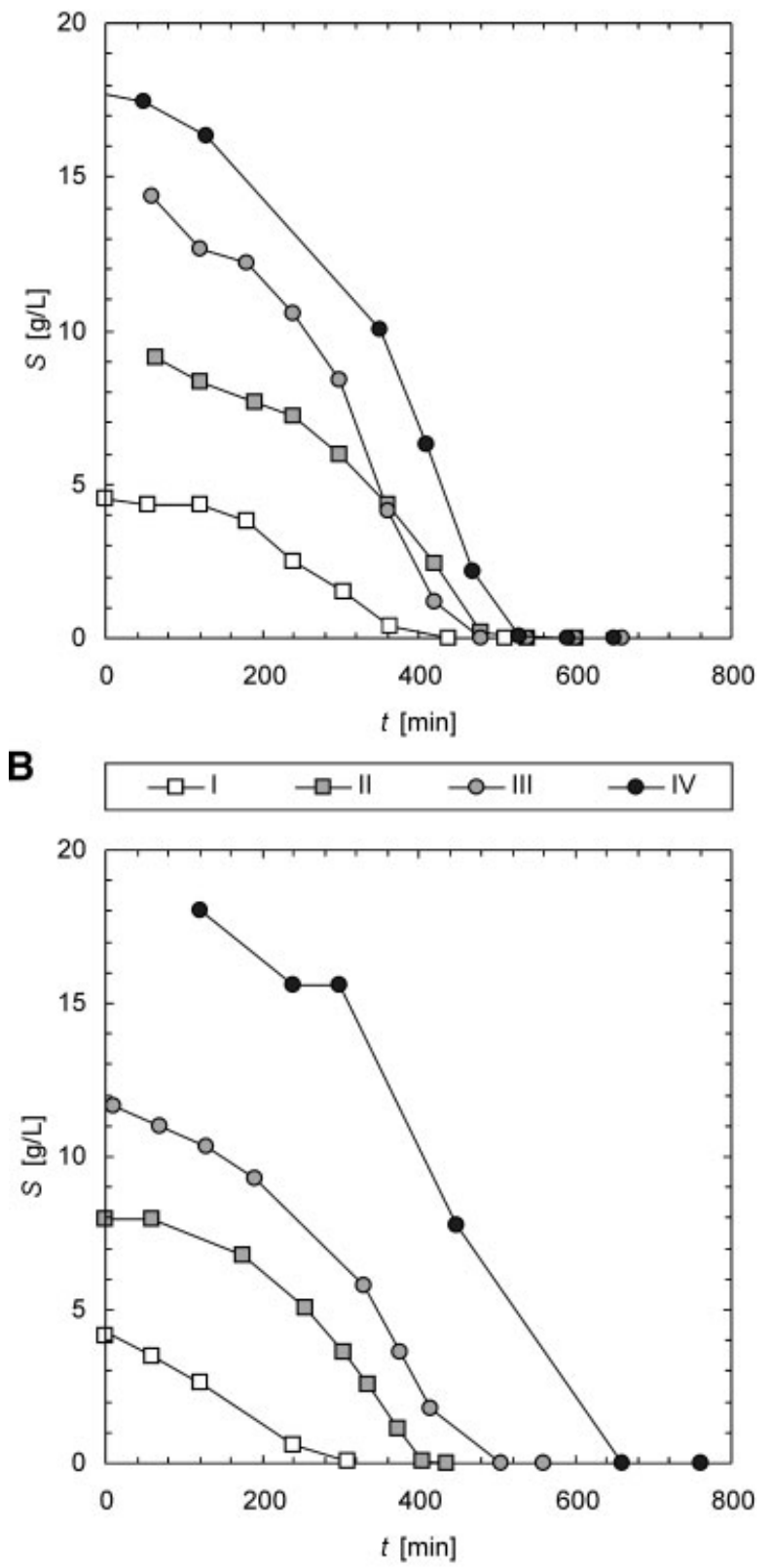

Figure 4. Time profiles of residual glucose concentrations $(S)$ in the aerobic batch growth on glucose of $S$. cerevisiae in bioprocesses I-IV (see Table I). Fermentations running in the 5-L ST bioreactor (A) and in the micro-bioreactor (B).

both ST and micro-bioreactor vessels. It may be suggested that, given the yields shown in Figure 6, the metabolism in the micro-bioreactor (with an average biomass production yield $=0.44$ ) is closer to pure-oxidative (with a typical average yield of 0.50) (Pamment et al., 1978).

\section{Substrate Consumption Kinetics}

Glucose can be metabolized both aerobically (through the oxidative pathway) and anaerobically (via the reductive or fermentative pathway), however, with different rates and different efficiencies (Sonnleitner and Kappeli, 1986). The

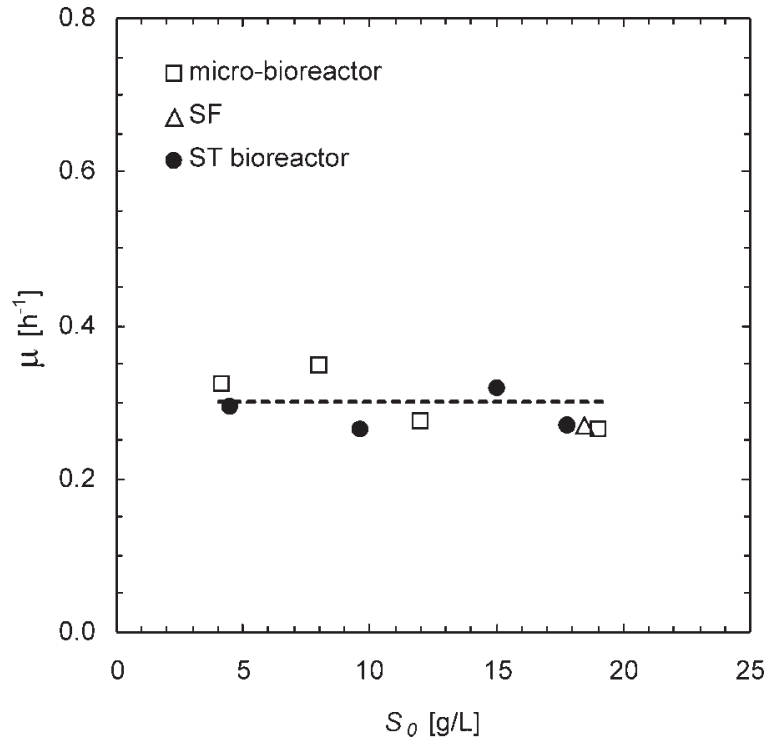

Figure 5. Specific growth rates $(\mu)$ for batch growth on glucose of $S$. cerevisiae at $25^{\circ} \mathrm{C}$ and different initial glucose concentrations $\left(S_{0}\right)$ in the 5-L ST bioreactor and in the micro-bioreactor. The specific growth rate presented for the SF was the averaged $\mu$ found for the seed culture growth, incubated at $27^{\circ} \mathrm{C}$ and $150 \mathrm{rpm}$.

yield coefficient $Y_{\mathrm{X} / \mathrm{S}}$ (on a mass basis) is the most reliable measurable (empirical) coefficient to summarize the substrate $(S)$ consumption for biomass $(X)$ formation. Typical values of $Y_{X / S}$ for $S$. cerevisiae are $0.47-0.50$ and $0.05-0.10$ for oxidative and reductive glucose breakdown pathways, respectively, at $T=30^{\circ} \mathrm{C}$ and $\mathrm{pH}=5.0$ (Sonnleitner and Kappeli, 1986). In the present work a global (oxidative plus reductive) $Y_{X / S}$ was determined for the exponential phase of yeast growth, as summarized in Table I. It is straightforward to conclude that higher $Y_{X / \mathrm{S}}$ values were obtained with the

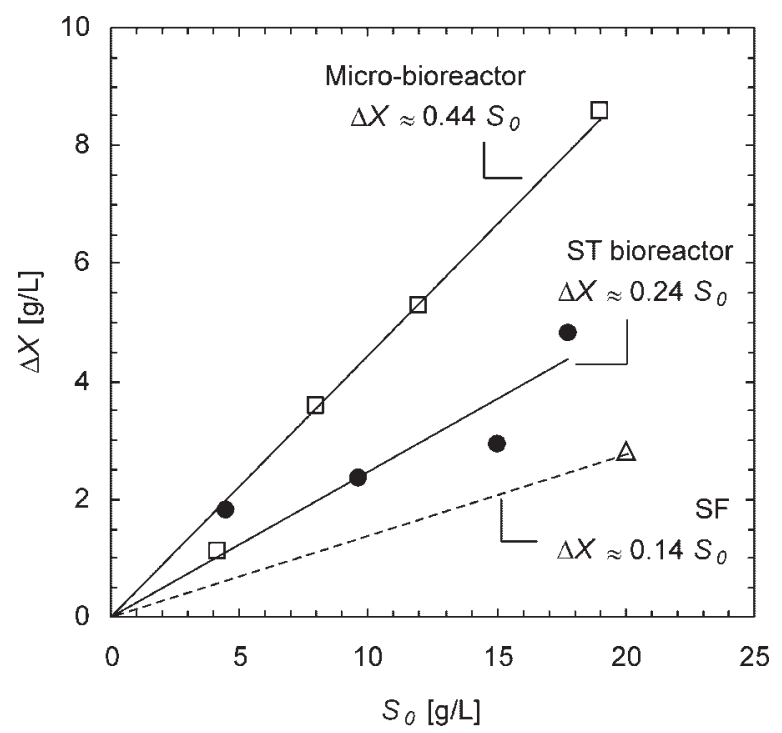

Figure 6. Increase in dry cell weight, $\Delta X=X-X_{0}$, obtained until complete depletion of glucose in the aerobic batch growth of $S$. cerevisiae on glucose in bioprocesses I-IV, for initial glucose concentrations $S_{0}$ of $\sim 5-20 \mathrm{~g} / \mathrm{L}$. 
micro-bioreactor. During the exponential growth, $Y_{X / S}$ was increased by up to $110 \%$ on the micro-bioreactor in comparison with the 5-L ST, also corresponding to more than a threefold increase in comparison with data from Haack et al. (2004). The $Y_{X / S}$ values for the fermentations in the micro-bioreactor are in all cases above $72 \%$ of the reported value $\left(Y_{X / S} \approx 0.50\right)$ for pure-oxidative (aerobic) growth of $S$. cerevisiae (Pamment et al., 1978), meaning that batch yeast growth in this reactor is achieved mostly through the respiratory pathway. Because respiration itself depends on the dissolved oxygen (Sonnleitner and Kappeli, 1986) such improvement on $Y_{X / S}$ values suggests enhanced dissolved oxygen availability in the micro-bioreactor due to the reciprocating nature of OFM. Also, the existence of inhomogeneities in the extremities of the ST vessel and its inherent oxygen gradients (Hadjiev et al., 2006) may be in the base for the lower performance of ST bioreactor.

The kinetics of substrate (glucose) consumption may be summarized in relation to the specific substrate uptake rate, $q_{\mathrm{s}}$ (Table I). Glucose flux was not differentiated between oxidative and reductive pathways, thus $q_{\mathrm{s}}$ represents the glucose uptake by both ways.

The batch aerobic growth of $S$. cerevisiae in the novel reactor presented lower values of $q_{\mathrm{s}}$ (30\% lower) in parallel with the higher $Y_{X / S}$ values, as compared with the ST. In both ST and micro-bioreactor systems the $S$. cerevisiae yeast is growing at a rate near $\mu_{\max }$ as shown previously. However, $q_{\mathrm{s}}$ is lower in the micro-bioreactor, thus the amount of glucose exceeding the bottleneck and being metabolized according to the reduction pathway is decreased. This results in enhanced
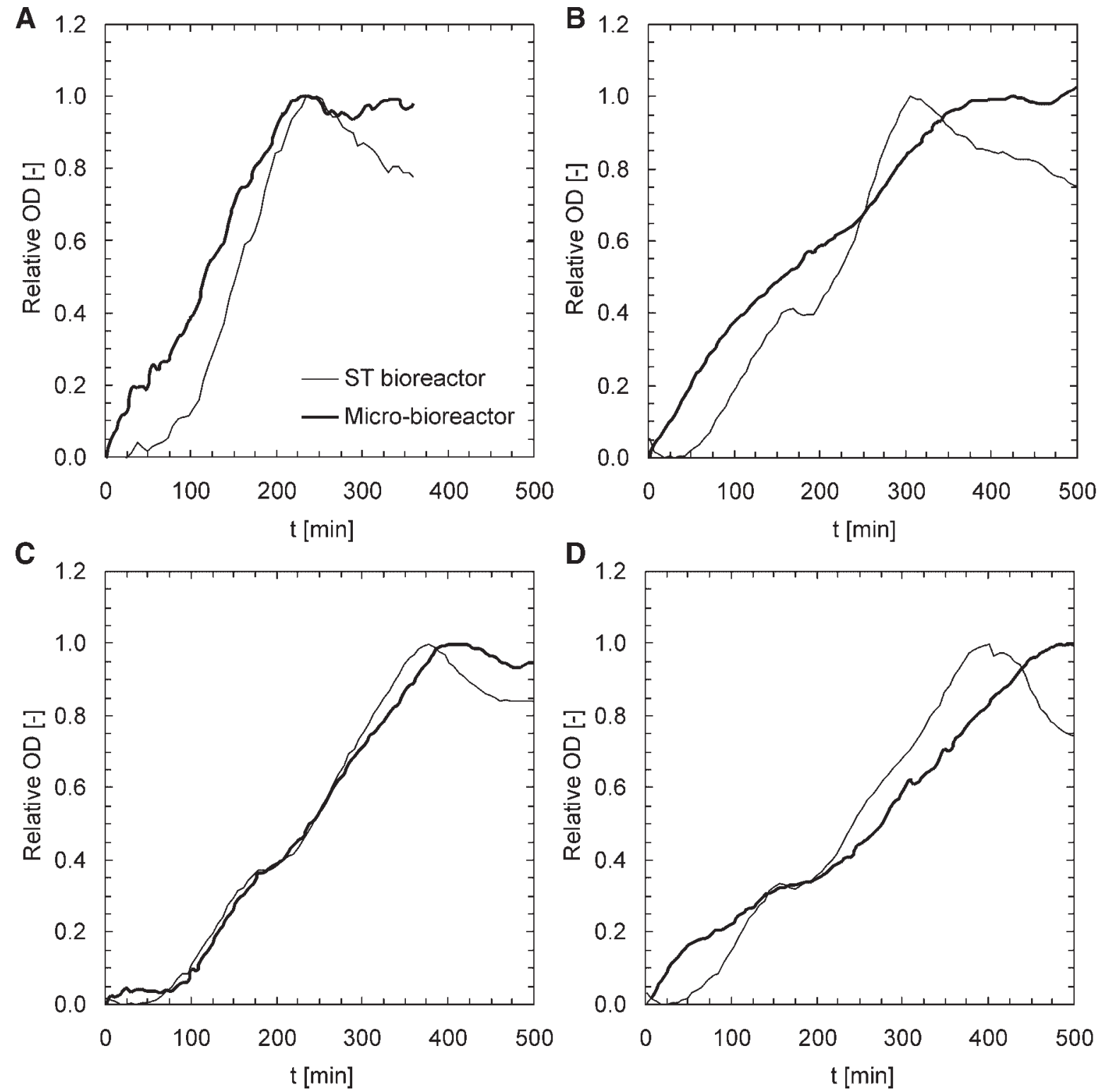

D

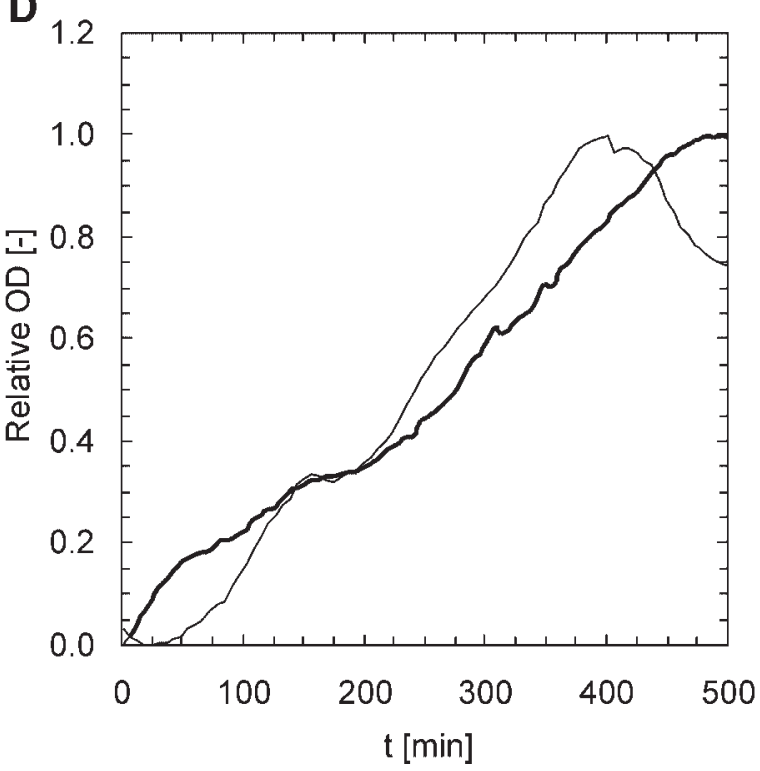

Figure 7. Time course of anaerobic batch growth on glucose (expressed as a relative function of the OD) of S. cerevisiae in the 2-L ST reactor and in the microbioreactor. Experiments were run in parallel and started with glucose concentrations of $5 \mathrm{~g} / \mathrm{L}$ (A), $10 \mathrm{~g} / \mathrm{L}$ (B), $15 \mathrm{~g} / \mathrm{L}$ (C), and $20 \mathrm{~g} / \mathrm{L}$ (D). No seed culture was prepared and fermentation temperature was controlled at $25^{\circ} \mathrm{C}$. Note that $\mathrm{OD}$ was turned dimensionless with the OD peak obtained at the end of growth phase (i.e., at the instant of glucose depletion, as indicated from $\mathrm{pH}$ measurements). 
biomass formation per unit of substrate consumed, that is, higher $Y_{X / S}$ values. In summary, the respiro-fermentative flux distribution of $S$. cerevisiae in ST bioreactor is redirected with the micro-bioreactor towards the oxidative pathway, allowing, for example, the optimization of industrial production of $S$. cerevisiae.

\section{Anaerobic Growth of $\boldsymbol{S}$. cerevisiae on Glucose}

Anaerobic growth of S. cerevisiae was monitored with the online and real-time OD of the culture media in both the 2-L ST and the micro-bioreactor using dip-optical reflectance probes, for initial glucose concentrations in the range 5$20 \mathrm{~g} / \mathrm{L}$. As no gas bubbles were present, a constant relation was obtained between the on-line OD signal and the biomass concentration.

Under anaerobic conditions, glucose is metabolized by the reductive (fermentative) pathway with the consequent formation of ethanol.

A comparison of batch anaerobic growth of $S$. cerevisiae for $S_{0}$ between 5 and $20 \mathrm{~g} / \mathrm{L}$ is presented in Figure 7 for both ST bioreactor and micro-bioreactor vessels. The OD increase along the fermentation time was very similar in both the 2-L ST and the micro-bioreactor, which means similar growth behavior of the yeast in these two vessels. However, in the 2-L ST bioreactor's experiments a decrease in OD was observed after the end of fermentation (OD peak), which was coincident with the observed biomass flocculation. This phenomenon is due to the glucose exhaustion: under these circumstances, the $S$. cerevisiae strain tends to flocculate, thus reducing the absorbance of the fermentation broth. In fact, the ideal brewing yeast (as the $S$. cerevisiae strain used in this work) grows as discrete cells and flocculates after sugar depletion from the media, leaving clear beer (Stewart and Russell, 1981). The differences found between ST and microbioreactor after glucose depletion mean that although stirred, the intensity of mixing provided by the $150 \mathrm{rpm}$ in the 2-L ST was insufficient to keep all the biomass suspended, while the micro-bioreactor proved to be a better device to keep cells in suspension.

\section{CONCLUSIONS}

As a proof-of-concept several aerobic fermentations were carried out in a novel micro-bioreactor system using as workhorse a flocculent $S$. cerevisiae strain at four different initial glucose concentrations $(\sim 5-20 \mathrm{~g} / \mathrm{L})$. Average $83 \%$ and $214 \%$ increases on biomass production were obtained with the micro-bioreactor when compared with a stirred-tank (ST) bioreactor and the seed culture growth in a shake flask (SF), respectively. It was postulated that the respirofermentative flux distribution of $S$. cerevisiae from the ST bioreactor was redirected in the micro-bioreactor towards the oxidative pathway. This was explained by the enhanced mass transfer rates achieved with this novel scaled-down platform, as demonstrated elsewhere (Reis et al., 2004a). Anaerobic fermentations at the same initial glucose concentration ranges $(5-20 \mathrm{~g} / \mathrm{L})$ gave the opportunity to use state-ofthe-art fiber optics technology for on-line and real-time monitoring/control of bioprocesses within this novel microbioreactor. A time profile of OD was observed to be very similar in the ST and in the micro-bioreactor, with a highly reproducible yeast growth in the two platforms.

The higher efficiencies of biomass production obtained as well as the much lower air-inlet requirements turn this novel micro-bioreactor also a potential platform for upstream development of animal cells derived bioprocesses. Moreover, the linear scale-up of reactors based on the OFM technology anticipates a parallel screening/optimization of bioprocesses with this novel micro-reactor at similar conditions to those at industrial scale. However, despite of the associated advantages, the very particular macroscopic flow patterns of OFM may pose additional difficulties when interpreting the results obtained with the system presented here in view of a future scale-up for different platforms (e.g., the STR). Future work will be focused in the development of a multiple-SPC tube unit allowing the high-throughput bioprocess optimization in a single unit volume.

Thanks are due to Fundação para a Ciência e a Tecnologia for financial support of co-author N. Reis through scholarship SFRH/BD/6954/ 2001.

\section{References}

Beck C, Vonmeyen HK. 1968. Enzyme pattern and aerobic growth of Saccharomyces cerevisiae under various degrees of glucose limitation. J Bacteriol 96(2):479-486.

Buchs J. 2001. Introduction to advantages and problems of shaken cultures. Biochem Eng J 7(2):91-98.

Doig SD, Pickering SCR, Lye GJ, Woodley JM. 2002. The use of microscale processing technologies for quantification of biocatalytic Baeyer-Villiger oxidation kinetics. Biotechnol Bioeng 80(1): 42-49.

Doig SD, Diep A, Baganz F. 2005a. Characterisation of a novel miniaturised bubble column bioreactor for high throughput cell cultivation. Biochem Eng J 23(2):97-105.

Doig SD, Ortiz-Ochoa K, Ward JM, Baganz F. 2005b. Characterization of oxygen transfer in miniature and lab-scale bubble column bioreactors and comparison of microbial growth performance based on constant k(L)alpha. Biotechnol Prog 21(4):1175-1182.

Duetz WA, Witholt B. 2001. Effectiveness of orbital shaking for the aeration of suspended bacterial cultures in square-deepwell microtiter plates. Biochem Eng J 7(2):113-115.

Duetz WA, Ruedi L, Hermann R, O’Connor K, Buchs J, Witholt B. 2000. Methods for intense aeration, growth, storage, and replication of bacterial strains in microtiter plates. Appl Environ Microbiol 66(6): 2641-2646.

Girard P, Jordan M, Tsao M, Wurm FM. 2001. Small-scale bioreactor system for process development and optimization. Biochem Eng J 7(2):117119.

Haack MB, Eliasson A, Olsson L. 2004. On-line cell mass monitoring of Saccharomyces cerevisiae cultivations by multi-wavelength fluorescence. J Biotechnol 114(1-2):199-208.

Hadjiev D, Sabiri NE, Zanati A. 2006. Mixing time in bioreactors under aerated conditions. Biochem Eng J 27(3):323-330. 
Kappeli O. 1986. Regulation of carbon metabolism in Saccharomyces Cerevisiae and related yeasts. Adv Microb Physiol 28:181-209.

Kostov Y, Harms P, Randers-Eichhorn L, Rao G. 2001. Low-cost microbioreactor for high-throughput bioprocessing. Biotechnol Bioeng 72(3):346-352.

Lamping SR, Zhang H, Allen B, Shamlou PA. 2003. Design of a prototype miniature bioreactor for high throughput automated bioprocessing. Chem Eng Sci 58(3-6):747-758.

Lee CT, Mackley MR, Stonestreet P, Middelberg APJ. 2001. Protein refolding in an oscillatory flow reactor. Biotechnol Lett 23(22):18991901.

Lee CT, Buswell AM, Middelberg APJ. 2002. The influence of mixing on lysozyme renaturation during refolding in an oscillatory flow and a stirred-tank reactor. Chem Eng Sci 57(10):1679-1684.

Li JC, Wang S, VanDusen WJ, Schultz LD, George HA, Herber WK, Chae HJ, Bentley WE, Rao G. 2000. Green fluorescent protein in Saccharomyces cerevisiae: Real-time studies of the GAL1 promoter. Biotechnol Bioeng 70(2):187-196.

Mackley MR. 1991. Process innovation using oscillatory flow within baffled tubes. Chem Eng Res Des 69(3):197-199.

Maier U, Buchs J. 2001. Characterisation of the gas-liquid mass transfer in shaking bioreactors. Biochem Eng J 7(2):99-106.

Marin MR. 1999. Alcoholic fermentation modelling: Current state and perspectives. Am J Enol Viticulture 50(2):166-178.

Ni XW, Gao SW, Pritchard DW. 1995. Study of mass-transfer in yeast in a pulsed baffled bioreactor. Biotechnol Bioeng 45(2):165-175.

Pamment NB, Hall RJ, Barford JP. 1978. Mathematical-modeling of lag phases in microbial-growth. Biotechnol Bioeng 20(3):349381.

Reis N, Vicente AA, Teixeira JA, Mackley MR. 2004a. Enhanced mass transfer rates of a novel oscillatory flow screening reactor. Summaries of the 16th International Congress of Chemical and Process Engineering; 22nd-26th August; Praha, Czech Republic. pp 898899.
Reis N, Vicente AA, Teixeira JA, Mackley MR. 2004b. Residence times and mixing of a novel continuous oscillatory flow screening reactor. Chem Eng Sci 59(22-23):4967-4974.

Reis N, Harvey AP, Vicente AA, Teixeira JA, Mackley MR. 2005. Fluid mechanics and design aspects of a novel oscillatory flow meso-reactor. Chem Eng Res Des 83(A4):357-371.

Rhodes RP, Gaden EL. 1957. Characterization of agitation effects in shaken flasks. Ind Eng Chem 49(8):1233-1236.

Rieger M, Kappeli O, Fiechter A. 1983. The role of limited respiration in the incomplete oxidation of glucose by Saccharomyces Cerevisiae. J Gen Microbiol 129:653-661.

Sonnleitner B, Kappeli O. 1986. Growth of Saccharomyces-cerevisiae is controlled by its limited respiratory capacity-Formulation and verification of a hypothesis. Biotechnol Bioeng 28:927-937.

Stewart GG, Russell I. 1981. Yeast flocculation. In: Pollock JRA, editor. Brewing science. Toronto: Academic Press. pp 61-92.

Tholudur A, Ramirez WF, McMillan JD. 1999. Mathematical modeling and optimization of cellulase protein production using trichoderma reesei RL-P37. Biotechnol Bioeng 66(1):1-16.

Vandijken JP, Scheffers WA. 1986. Redox balances in the metabolism of sugars by yeasts. Fems Microbiol Rev 32(3-4):199-224.

Verduyn C, Zomerdijk TPL, Vandijken JP, Scheffers WA. 1984. Continuous measurement of ethanol-production by aerobic yeast suspensions with an Enzyme Electrode. App Microbiol Biotechnol 19(3):181-185.

Walther I, Vanderschoot BH, Jeanneret S, Arquint P, Derooij NF, Gass V, Bechler B, Lorenzi G, Cogoli A. 1994. Development of a miniature bioreactor for continuous-culture in a space laboratory. J Biotechnol 38(1):21-32.

Weiss S, John GT, Klimant I, Heinzle E. 2002. Modeling of mixing in 96-well microplates observed with fluorescence indicators. Biotechnol Prog 18(4):821-830.

Yu ZS, Zhang HX. 2004. Ethanol fermentation of acid-hydrolyzed cellulosic pyrolysate with Saccharomyces cerevisiae. Bioresource Technol 93(2): 199-204. 